\title{
QUANTUM ENTANGLEMENT IN ONE-DIMENSIONAL ANYONS
}

\author{
H S Mani, Ramadas N, V V Sreedhar \\ Chennai Mathematical Institute, SIPCOT IT Park, Siruseri, Chennai, 603103 India \\ hsmani@cmi.ac.in, ramadasn@cmi.ac.in, sreedhar@cmi.ac.in
}

\begin{abstract}
Anyons in one spatial dimension can be defined by correctly identifying the configuration space of indistinguishable particles and imposing Robin boundary conditions. This allows an interpolation between the bosonic and fermionic limits. In this paper, we study the quantum entanglement between two one-dimensional anyons on a real line as a function of their statistics.
\end{abstract}

\section{Introduction}

It is well-known that, in quantum mechanics, the indistinguishability of particles forces the multiparticle wave functions to be either symmetric (bosonic) or antisymmetric (fermionic) under the exchange of any pair of particles. In the last few decades it has emerged that in low dimensions it is possible to have more general quantum statistics. The classical roots for this can be traced to the non-trivial topology of the associated configuration space. Particles which obey these generalised statistics are called anyons, and they interpolate between bosons and fermions. Interestingly, these particles appear as collective excitations in fractional quantum Hall systems. In view of this, the quantum mechanical and thermodynamic properties of anyons have been extensively studied [1] [2].

The interest in anyons has been revived recently because of their potential application in topological quantum computation [3. In topological quan- 
tum computation, instead of using qubits one uses anyons to store information in their non-trivial wave functions. Since these are topologically protected, it is hoped that a topological quantum computer leads to fault-tolerant and decoherence-free computation [4] [5].

However, a completely robust, fault-tolerant physical system is not desirable because it does not allow us to store any information, let alone manipulate or extract it. In view of this, it is important to allow the system to interact with the apparatus (environment) in a controlled manner.

This motivates us to revisit the old problems of anyon quantum mechanics, and study them in the framework of open quantum systems. In particular, we are interested in knowing how the entanglement between two anyons depends on the statistics parameter when one of them is considered to be the system, and the other, the environment.

There are two complexities associated with this problem. First, it is wellknown that for indistinguishable particles, the standard methods used to quantify the entanglement, like finding the Schmidt rank, taking a partial trace, and finding the von Neumann entropy fail to work. The main reason for this is the non-factorizability of the multi-particle Hilbert space of indistinguishable particles. Various approaches has been proposed to circumvent this problem [6] [7] [9] 10] 11] 12] [13][14. Second, these approaches mostly restrict their attention to bosons and fermions.

Returning to our problem, we find it useful to follow the information theoretic approach to quantum entanglement developed by Lo Franco and Compagno [10]. In their work they show how it is possible to define the reduced density matrix in a system of indistinguishable particles by defining an inner product between states belonging to Hilbert spaces with different dimensionalities. It is straightforward to recast this method in the language of second quantization [15] [16], which is especially suited for our purposes. Within this framework, we show how the results can be generalised to anyons by the simple prescription of using the anyonic algebra for the creation and annihilation operators instead of the bosonic and fermionic algebras which are recovered as special cases. 
The rest of the paper is organized as follows.

In section 2, we review the information theoretic approach developed by Lo Franco and Compagno, with special emphasis on its reformulation in the language of second quantization.

In section 3, we review the model of indistinguishable particles on a real line, first studied by Leinaas and Myrheim [17. In this model they first construct the classical configuration space by identifying different configurations which can be obtained by permutations of particle positions, and then quantize the system to obtain a wave function that interpolates between the bosonic and fermionic limits through a statistics parameter $\eta$ coming from the Robin boundary conditions. A second quantization of this model [18 gives rise to an $\eta$-dependent algebra for the creation and annihilation operators, which reduces to the usual bosonic and fermionic algebras as limiting cases.

In section 4 , we use the above results to compute the reduced density matrix and the von Neumann entropy of a system of two anyons on a line.

In section 5 we conclude by giving a summary and an outlook.

\section{Information Theoretic Approach to Indistin- guishable Particles}

In the usual approach, a state of a system of indistinguishable particles is obtained by first quantizing the system as if the particles were distinguishable, by labelling them. We then apply the symmetrization postulate on the product wave functions to get bosonic and fermionic states [19].

It is instructive to restate this in the language of transition amplitudes. For example, a two-particle state is simply written as $|\psi, \phi\rangle$, where $\psi$ and $\phi$ represent single particle states. For indistinguishable particles, this two-particle state should be thought of as a holistic entity; it is not possible to say which particle is in which single particle state. Since the particles are not labelled, it is evident that the symmetrization postulate is not invoked. Quantum statistics 
enters through the definition of the inner product of these states.

For distinguishable particles, an initial state $|\phi, \psi\rangle$ can only evolve into the final state, say, $|\varphi, \zeta\rangle$ for which we compute the amplitude. But when the particles are indistinguishable, both the final states $|\varphi, \zeta\rangle$ and $|\zeta, \varphi\rangle$ contribute to the amplitude. For the case of bosons and fermions, the simple recipe of introducing the right sign to account for the exchange takes care of this complication.

This ad hoc procedure does not easily generalise to anyons. It is therefore desirable to have a more fundamental approach to the problem where the indistinguishability of the particles is maintained through out. This is the idea behind the information theoretic approach developed in [10.

If $|\varphi, \zeta\rangle$ and $|\phi, \psi\rangle$ denote two two-particle states, their inner product is,

$$
\langle\varphi, \zeta \mid \phi, \psi\rangle=\langle\varphi \mid \phi\rangle\langle\zeta \mid \psi\rangle+\eta\langle\varphi \mid \psi\rangle\langle\zeta \mid \phi\rangle .
$$

where $\eta=1$ for bosons and $\eta=-1$ for fermions.

The inner product between states belonging to Hilbert spaces of different dimensionality can also be defined. If we consider an unnormalized two-particle state, $|\Phi\rangle=\left|\varphi_{1}, \varphi_{2}\right\rangle$, the inner product with a single-particle state $|\psi\rangle$ is

$$
\left\langle\psi|\cdot| \varphi_{1}, \varphi_{2}\right\rangle \equiv\left\langle\psi \mid \varphi_{1}, \varphi_{2}\right\rangle=\left\langle\psi \mid \varphi_{1}\right\rangle\left|\varphi_{2}\right\rangle+\eta\left\langle\psi \mid \varphi_{2}\right\rangle\left|\varphi_{1}\right\rangle
$$

This is a projective measurement on a single particle, where the unnormalized two-particle state is projected on to $|\psi\rangle$. In a similar manner, the inner product between an $N$-particle state and a single-particle state is also defined. This definition of inner product between states belonging to Hilbert spaces with different dimensions can be used to define the reduced density matrix as shown below.

Let $|\Phi\rangle$ be a normalized $N$-particle state. To perform the partial trace we choose a basis $\left\{\left|\psi_{k}\right\rangle\right\}$ for the single-particle Hilbert space. The normalized pure state after projecting on to a state $\left|\psi_{k}\right\rangle$ is

$$
\left|\phi_{k}\right\rangle=\frac{\left\langle\psi_{k} \mid \varphi_{1}, \varphi_{2}\right\rangle}{\sqrt{\left\langle\Pi_{k}^{(1)}\right\rangle_{\Phi}}}
$$

where $\Pi_{k}^{(1)}=\left|\psi_{k}\right\rangle\left\langle\psi_{k}\right|$. 
Define a one-particle identity operator as $\mathbb{I}^{(1)}=\sum_{k} \Pi_{k}^{(1)}$. Then the probability of finding a single particle in the state $\left|\psi_{k}\right\rangle$ is

$$
p_{k}=\frac{\left\langle\Pi_{k}^{(1)}\right\rangle_{\Phi}}{\left\langle\mathbb{I}^{(1)}\right\rangle_{\Phi}}
$$

With the knowledge of $\left|\phi_{k}\right\rangle$ and the corresponding probabilities $p_{k}$, the reduced density matrix is defined as follows

$$
\rho^{(1)}=\operatorname{Tr}^{(1)}|\Phi\rangle\left\langle\Phi\left|=\sum_{k} p_{k}\right| \phi_{k}\right\rangle\left\langle\phi_{k}\right|
$$

After obtaining the reduced density matrix, the von Neumann entropy can be calculated as usual,

$$
S\left(\rho^{(1)}\right)=-\operatorname{Tr}\left(\rho^{(1)} \log \rho^{(1)}\right)=-\sum_{i} \lambda_{i} \log \lambda_{i}
$$

where $\lambda_{i}$ is an eigenvalue of the reduced density matrix.

\section{Second quantization formalism}

We can recast the above idea in the language of second quantization. If $|\Phi\rangle$ is an $N$-particle state, its inner product with a single-particle state $\left|\psi_{k}\right\rangle$ is [15]

$$
a_{\psi_{k}}|\Phi\rangle \equiv\left\langle\psi_{k}|\cdot| \Phi\right\rangle
$$

Note that since $a_{\psi_{k}}$ is an annihilation operator, the left hand side of the above equation represents an $(N-1)$-particle state which, by definition, is the inner product on the right hand side. As mentioned earlier, this simple expedient allows us to go beyond bosons and fermions by suitably generalising the operator algebra. We present this in the next section.

We conclude this section by noting that the expression for the reduced density matrix in the second quantization formalism is

$$
\rho^{(1)}=\operatorname{Tr}^{(1)}|\Phi\rangle\langle\Phi|=\frac{\sum_{k} a_{\psi_{k}}|\Phi\rangle\langle\Phi| a_{\psi_{k}}^{\dagger}}{\langle\Phi|\hat{\mathbf{n}}| \Phi\rangle}
$$

Here $\hat{\mathbf{n}}=\sum_{k} a_{\psi_{k}}^{\dagger} a_{\psi_{k}}$ is the total number operator. The details are given in appendix $\mathrm{A}$ 


\section{Anyons}

It is well-known that, in relativistic quantum field theory, the spin-statistics theorem [20] dictates that bosonic fields satisfy canonical commutation relations, while fermionic fields satisfy anti-commutation relations. In nonrelativistic quantum mechanics, one mimics the quantum field theoretic ideas through second quantization which directly yields multi-particle wave functions of indistinguishable particles with appropriate symmetry properties. In particular, particles with (half-)integer spin have wave functions which are (anti-)symmetric under the exchange of any two particles.

In contrast, the Symmetrization Postulate [19] accomplishes this objective by attaching labels to the particles, as if they were distinguishable, and (anti)symmetrizing the product wave function with respect to these labels. But, labelling indistinguishable particles is intrinsically contradictory. So, it is desirable to look beyond this ad hoc prescription.

In a seminal paper, Leinaas and Myrheim [17] trace the origin of the Symmetrization Postulate to the non-trivial topology of the underlying classical configuration space of indistinguishable particles. As a spin-off of this insight, they show that, in low dimensions, it is possible to have objects which are more general than bosons and fermions. These are called anyons. In what follows, we briefly summarise the Leinaas-Myrheim method that leads to anyons.

Let us consider a system of $N$ spin-less particles in $d$ dimensions. Let $X=\mathbf{R}^{d}$ be the configuration space of a single particle. If the particles are distinguishable, the configuration space of the system is $\mathcal{X}_{N}=X^{N}$ where $X^{N}$ denotes an $N$ - fold tensor product of the single-particle space $X$. A point in the space $\mathbf{x}=\left(x_{1}, x_{2}, \ldots, x_{N}\right)$ represents a physical configuration of the system.

If the $N$ particles are indistinguishable, the configuration space is $\mathcal{Y}_{N}=$ $\left(X^{N}-D\right) / S_{N}$ where $S_{N}$ is the permutation group on $N$ elements. It ensures that the points $\mathbf{x}=\left(x_{1}, x_{2}, \ldots, x_{N}\right)$ and $\mathbf{x}^{\prime}=\left(x_{P(1)}, x_{P(2)}, \ldots, x_{P(N)}\right)$ which represent the same physical configuration are identified. Here $P$ represents an arbitrary permutation. $D$ represents the set of singular points which are 
unaffected by the identifications.

In the above, the description is entirely classical. The idea is that since the identifications have been made already at the level of the classical configuration space, the restrictions on quantum states would follow without the ad hoc need to invoke the symmetrization postulate. For particles with spin, one continues to define the configuration space as above, with the minor modification that at each point in $\mathcal{Y}_{N}$ we erect a spinor space. The spin observables act as operators on this spinor space. We refer the reader to [17] for further details.

In the above formalism, the quantum mechanical wave function of the system is determined by the one-dimensional unitary representations of the fundamental group $\pi_{1}\left(\mathcal{Y}_{N}\right)$ of the configuration space. For the case of indistinguishable particles, this turns out to be the permutation group in dimensions $d \geq 3$, whose lowest dimensional irreducible representations allow only bosons and fermions. In two dimensions, the fundamental group of the system is $\pi_{1}\left(\mathcal{Y}_{N}\right)=B_{N}$, where $B_{N}$ is the braid group on $N$ strings, whose one dimensional unitary representations allow the wave function to pick up a phase $e^{i \theta}$, where $\theta$ is a real parameter, under an exchange. This is the underlying reason for the possibility of having anyons in low dimensions.

\section{Indistinguishable Particles On the Real Line}

In the case of indistinguishable particles on a real line, it is not possible to perform an exchange without taking the particles through each other: an exchange gets inextricably linked with scattering. It is neverthless possible to define quantum statistics by following the Leinaas-Myrheim prescription, as shown below in the specific example of two indistinguishable particles on a real line. If $x_{1}$ and $x_{2}$ are the positions of the particles, we observe that the points $\mathbf{x}=\left(x_{1}, x_{2}\right)$ and $\mathbf{x}^{\prime}=\left(x_{2}, x_{1}\right)$ represent the same configuration, and hence need to be identified. The identification is done by folding the $\left(x_{1} x_{2}\right)$ plane along the line $x_{1}=x_{2}$ which represents the singular points. Without loss of generality, we choose to work with the half plane $x_{1}<x_{2}$. The problem can be solved by prescribing 
appropriate boundary conditions along the diagonal.

We choose the free particle Hamiltonian for the system, also studied by Posske et al [21],

$$
H=-\frac{1}{2}\left(\frac{\partial^{2}}{\partial x_{1}^{2}}+\frac{\partial^{2}}{\partial x_{2}^{2}}\right)
$$

where we use the units $\hbar=c=1$ and set mass equal to one. To ensure that particles remain bounded in the region $x_{1}<x_{2}$, we impose the boundary condition that the normal component of the probability current vanishes at the boundary. That is,

$$
\left.\left(\psi^{*}(\mathbf{x})\left(-\frac{\partial}{\partial x_{1}}+\frac{\partial}{\partial x_{2}}\right) \psi(\mathbf{x})-\psi(\mathbf{x})\left(-\frac{\partial}{\partial x_{1}}+\frac{\partial}{\partial x_{2}}\right) \psi^{*}(\mathbf{x})\right)\right|_{x_{1}=x_{2}}=0
$$

Note that above equation also ensures self-adjointness of the Hamiltonian. The general solution of the above equation is given by,

$$
\left.\left(-\frac{\partial}{\partial x_{1}}+\frac{\partial}{\partial x_{2}}\right) \psi(\mathbf{x})\right|_{x_{1}=x_{2}}=\left.\eta \psi(\mathbf{x})\right|_{x_{1}=x_{2}}
$$

where $\eta$ is a real parameter. The eigenstates of the Hamiltonian are

$$
\psi(\mathbf{x})=e^{i\left(k_{1} x_{1}+k_{2} x_{2}\right)}+e^{-i\left(\phi_{\eta}\left(k_{2}-k_{1}\right)\right)} e^{i\left(k_{2} x_{1}+k_{1} x_{2}\right)}
$$

where,

$$
\phi_{\eta}\left(k_{2}-k_{1}\right)=2 \tan ^{-1}\left(\frac{\eta}{k_{2}-k_{1}}\right)
$$

Note that $\eta=0$ and $\eta=\infty$ correspond to Neumann and Dirichlet boundary conditions respectively on the diagonal i.e. the set of coincident points $x_{1}=x_{2}$. The former gives a symmetric wave function, while the latter gives an antisymmetric wave function which also enforces the Pauli Exclusion Principle. Arbitrary values of $\eta$ correspond to Robin boundary conditions, with the corresponding wave functions being neither symmetric nor antisymmetric. These are, by definition, one-dimensional anyons.

For $\eta<0$, it is easy to see that the system admits one bound state 1 This follows from the requirement that the wave function is well-behaved at $\pm \infty$,

\footnotetext{
${ }^{1}$ The Hamitonian, despite its appearance, it is not positive definite because of the boundary. This is what allows for the existence of a bound state.
} 
which in turn implies that the momentum of the centre of mass coordinate is purely real, and the momentum of the relative coordinate is purely imaginary.

We mention in passing that for the case of three or more particles, there are several diagonals corresponding to coincident points; but the Robin boundary conditions can be generalized in a straightforward manner as shown in the next subsection.

\section{$N$ particles on the real line}

In the case of $N$ identical particles on a real line the configuration space can be constructed in a similar way and is chosen to be the region where $\mathcal{R}=\left\{\mathbf{x} \mid x_{1}<\right.$ $\left.x_{2}<x_{3}<\ldots<x_{N}\right\}$. The Hamiltonian is again the free particle Hamiltonian

$$
H=-\frac{1}{2} \sum_{j=1}^{N} \frac{\partial^{2}}{\partial x_{j}^{2}}
$$

and the Robin boundary conditions are

$$
\left.\left(\frac{\partial}{\partial x_{j+1}}-\frac{\partial}{\partial x_{j}}\right) \psi(\mathbf{x})\right|_{x_{j+1}=x_{j}}=\left.\eta \psi(\mathbf{x})\right|_{x_{j+1}=x_{j}}
$$

The corresponding anyonic wave functions are obtained by solving the Schrodinger equation for which we employ the ansatz $\psi(\mathbf{x})=\int_{\mathbf{k} \in \mathbb{C}^{n}} d \mathbf{k} \alpha(\mathbf{k}) e^{i \mathbf{k x}}$. The coefficients $\alpha(k)$ satisfy,

$$
\alpha(\mathbf{k})=\left\{\begin{array}{l}
e^{-i\left(\phi_{\eta}\left(k_{j+1}-k_{j}\right)\right)} \alpha\left(P_{j} \mathbf{k}\right) \text { if } k_{j+1}-k_{j} \neq i \eta \\
0 \text { if } k_{j+1}-k_{j}=i \eta
\end{array}\right.
$$

where an elementary permutation $P_{j}$ permutes the $j$ th and $(j+1)$ th elements and

$$
\phi_{\eta}\left(k_{j+1}-k_{j}\right)=2 \tan ^{-1}\left(\frac{\eta}{k_{j+1}-k_{j}}\right)
$$

The connection between the coefficients can be written as follows

$$
\alpha(\mathbf{k})=e^{i \phi_{\eta}^{P}(\mathbf{k})} \alpha(P \mathbf{k})
$$

where $P=P_{j_{1}} \ldots . . P_{j_{r}}$ represents the minimum number of elementary permutations required to reach a given permutation.

$$
\phi_{\eta}^{P}(\mathbf{k})=\sum_{i=1}^{r} \phi_{\eta}\left[\left(P_{j_{1}} \ldots . . P_{j_{i}} \mathbf{k}\right)_{j_{i}}-\left(P_{j_{1}} \ldots . . P_{j_{i}} \mathbf{k}\right)_{j_{i+1}}\right] .
$$


The basis functions are of the form $\psi_{\mathbf{k}}(\mathbf{x}) \propto \sum_{P \in S_{n}} e^{i \phi_{\eta}^{P}(\mathbf{k})} e^{i(P \mathbf{k}) \mathbf{x}}$. As in the two-particle case, only special values of $\mathbf{k}$ are permitted when $\eta<0$. In contrast to the two-particle case, however, we can have bound states with different number of particles.

\section{Second quantization}

As already mentioned in the Introduction, we find it useful to recast the above results in the language of second quantization, as was done in [18. We use the following generalised $\eta$-dependent algebra for the second quantized creation operator $\Psi^{\dagger}(x)$, and annihilation operator $\Psi(x)$ of the anyon fields

$$
\begin{aligned}
{\left[\Psi(x), \Psi^{\dagger}(y)\right] } & =\delta(x-y)-2 \eta \int_{0}^{\infty} d z e^{-z \eta} \Psi^{\dagger}(y-z) \Psi(x-z) \\
{\left[\Psi^{\dagger}(x), \Psi^{\dagger}(y)\right] } & =-2 \eta \int_{0}^{\infty} d z e^{-z \eta} \Psi^{\dagger}(y+z) \Psi^{\dagger}(x-z) .
\end{aligned}
$$

Note that this algebra reduces to the standard bosonic and fermionic limits for $\eta \rightarrow 0$ and $\eta \rightarrow \infty$ respectively. Also note that this algebra is slightly different from the one presented in [18. As shown in appendix B.1, the above equations can be derived starting from the corresponding algebra for the creation and annihilation operators for momentum states, related to the second quantized fields through the usual relations $\Psi^{\dagger}(x)=\frac{1}{\sqrt{2 \pi}} \int_{-\infty}^{\infty} d k e^{i k x} a_{k}^{\dagger}$.

The following commutators involving the number operator $\hat{N}$ defined in the usual manner as $\hat{N}=\int_{-\infty}^{\infty} d x \Psi^{\dagger}(x) \Psi(x)$, can be derived in a straightforward manner as shown in appendix B.2

$$
\begin{aligned}
{\left[\hat{N}, \Psi^{\dagger}(y)\right] } & =\Psi^{\dagger}(y) \\
{[\hat{N}, \Psi(y)] } & =-\Psi(y)
\end{aligned}
$$

Thus, although the algebra for the anyonic fields is more complicated than the bosonic and fermionic cases, the number operator can be defined in the usual fashion, and satisfies the standard commutation relation with the second quantized fields. This allows us to interpret the matrix elements of the fields in the number operator basis as operators which transform multiparticle wave functions into other wave functions with more or fewer number of particles as 
explained by Fock 22 . In appendix B.3, we explicitly verify that the modified algebra satisfies the conditions derived by Fock.

\section{Entropy of Two Identical Particles}

We consider two indistinguishable particles on the real line. We assume that the statistics parameter $\eta$ is non-negative, so that the particles are anyons. Note that the bosonic and fermionic limits can be retrieved from the general case as special cases.

The field operator $\Psi^{\dagger}(x)$ acting on the vacuum creates a particle localised at $x$. Rather than dealing with these localised states, it is convenient for our purposes to work with smeared fields defined as follows: $\Psi_{f}^{\dagger}=\int_{-\infty}^{\infty} d x f(x) \Psi^{\dagger}(x)$, where $f(x) \in \mathcal{S}(\mathbb{R})$, is a function in the Schwartz space [23]. The algebra of the smeared fields is readily obtained to be

$$
\begin{aligned}
& {\left[\Psi_{f}, \Psi_{g}^{\dagger}\right]=\langle f \mid g\rangle-2 \eta \int_{0}^{\infty} d z \int_{-\infty}^{\infty} \int_{-\infty}^{\infty} d x d y f^{*}(x) g(y) e^{-z \eta} \Psi^{\dagger}(y-z) \Psi(x-z)} \\
& {\left[\Psi_{f}^{\dagger}, \Psi_{g}^{\dagger}\right]=-2 \eta \int_{0}^{\infty} d z \int_{-\infty}^{\infty} \int_{-\infty}^{\infty} d x d y f^{*}(x) g(y) e^{-z \eta} \Psi^{\dagger}(y+z) \Psi^{\dagger}(x-z)}
\end{aligned}
$$

where the inner product $\langle f \mid g\rangle=\int_{-\infty}^{\infty} d x f^{*}(x) g(x)$. We use the following notation to denote the states $|f\rangle \equiv \Psi_{f}^{\dagger}|0\rangle$. If we choose a set of orthonormal functions $\left\{f_{n}(x)\right\}$, the corresponding set of states $\left\{\left|f_{n}\right\rangle\right\}$ will form a basis for the single-particle Hilbert space. For our purpose we chose $f_{n}(x)=h_{n}(x)$, where $h_{n}(x)=\frac{1}{\sqrt{\sqrt{\pi} 2^{n} n !}} H_{n}(x) e^{-\frac{x^{2}}{2}}$ is $n$-th eigenstate of the harmonic oscillator.

Let the two-particle state be

$$
\left|\Phi_{j, i}\right\rangle \equiv \frac{1}{\mathcal{N}} \Psi_{h_{j}}^{\dagger} \Psi_{h_{i}}^{\dagger}|0\rangle
$$

Here $\mathcal{N}=\left\langle 0\left|\Psi_{h_{i}} \Psi_{h_{j}} \Psi_{h_{j}}^{\dagger} \Psi_{h_{i}}^{\dagger}\right| 0\right\rangle$ is the normalization constant. We use the oneparticle basis $\left\{\left|h_{n}\right\rangle\right\}$ as the basis to calculate both the partial trace and the eigenvalues of the reduced density matrix. The one-particle reduced density matrix $\rho^{(1)}$ is obtained from the two-particle state as follows

$$
\rho^{(1)}=\frac{\sum_{k=0}^{\infty} \Psi_{h_{k}}\left|\Phi_{j, i}\right\rangle\left\langle\Phi_{j, i}\right| \Psi_{h_{k}}^{\dagger}}{\left\langle\Phi_{j, i}|\hat{\mathbf{n}}| \Phi_{j, i}\right\rangle}
$$


where $\hat{\mathbf{n}}=\sum_{k=0}^{\infty} \Psi_{h_{k}}^{\dagger} \Psi_{h_{k}}$ is the total number operator. A matrix element of the reduced density matrix is given by

$$
\rho_{m, n}^{(1)}=\left\langle h_{m}\left|\rho^{(1)}\right| h_{n}\right\rangle=\frac{\sum_{k=0}^{\infty}\left\langle 0\left|\Psi_{h_{m}} \Psi_{h_{k}}\right| \Phi_{j, i}\right\rangle\left\langle\Phi_{j, i}\left|\Psi_{h_{k}}^{\dagger} \Psi_{h_{n}}\right| 0\right\rangle}{\left\langle\Phi_{j, i}|\hat{\mathbf{n}}| \Phi_{j, i}\right\rangle}
$$

The expressions for the matrix element can be obtained analytically. They are given by an infinite series involving parabolic cylinder functions. They depend on $\eta$. The detailed calculations are given in appendix C

Since the expressions for the reduced density matrix are cumbersome, we resort to calculating the eigenvalues numerically, by using the formula

$$
\sum_{m=0}^{\infty} \rho_{m, n}^{(1)} g(n)=\lambda_{n} g(m)
$$

where $\lambda_{n}$ is an eigenvalue. The von Neumann entropy is then given by the usual formula

$$
S\left(\rho^{1}\right)=-\operatorname{Tr}\left(\rho^{1} \log \left(\rho^{1}\right)\right)=-\sum_{i} \lambda_{i} \log \left(\lambda_{i}\right)
$$

The dependence of the von Neumann entropy on the statistics parameter $\eta$ is plotted in the following figures for different choices of the initial two-particle state.

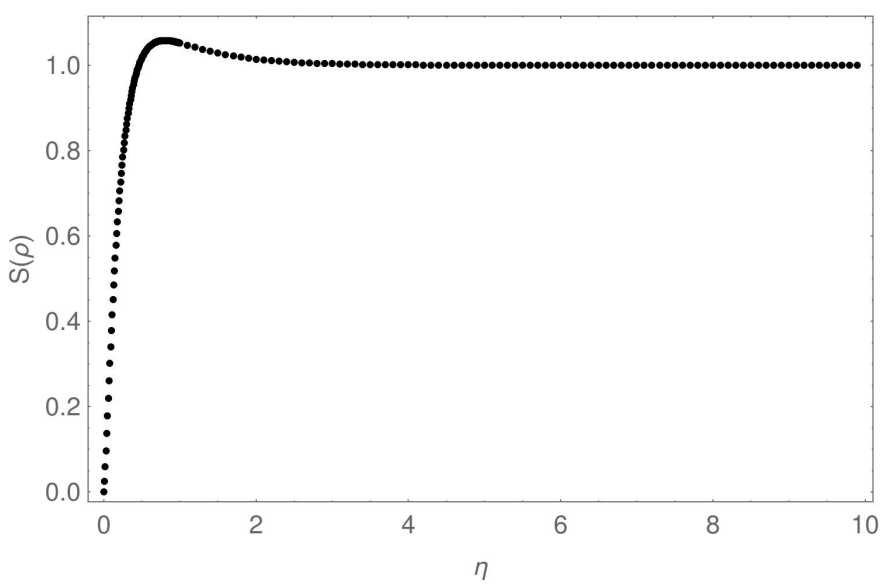

Figure 1: Plot of entropy vs statistics parameter $\eta$ for the initial two-particle state $\left|\Phi_{0,0}\right\rangle$. 
In the above plot, the two-particle state is taken to be $\left|\Phi_{0,0}\right\rangle$. It is worth noting that for $\eta=0$, both the particles are in the same state. The entropy is zero, consistent with what is expected of bosons. Note, however, that this plot is not valid in the fermionic limit $\eta \rightarrow \infty$, because the state $\left|\Phi_{0,0}\right\rangle$ identically vanishes as can be easily seen from equations 18,19 .

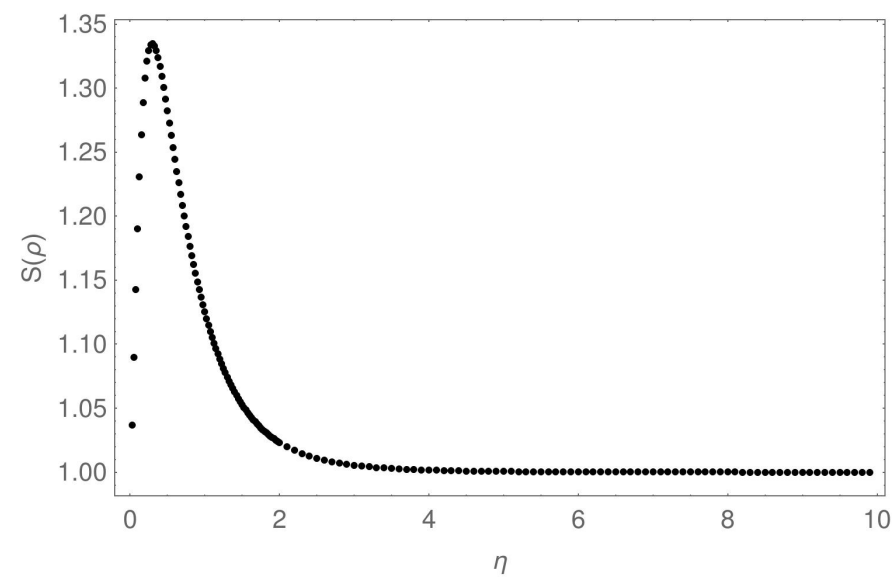

Figure 2: Plot of entropy vs statistics parameter $\eta$ for the initial two-particle state $\left|\Phi_{1,0}\right\rangle$.

In the above plot, the two-particle state is taken to be $\left|\Phi_{1,0}\right\rangle$. In this case, it worth noting that for both $\eta=0$ and $\eta \rightarrow \infty$, the entropy is equal to unity.

In order to get a better insight into what the above plots mean, it is useful to compare our results with [10. Lo Franco and Compagno consider a model of two indistinguishable qubits in an asymmetric double-well potential. In particular, they study the spin correlations between the qubits in the same spatially localised state, namely the left trough. It is important to note that the potential acts as a crutch to produce various states for the qubits, namely, states which are localised either on the left side, or the right side, or those which are in a superposition of the left and right sides. Once a state is specified, only the finitedimensional Hilbert spaces associated with the qubits play a role. For example, they show that when both the qubits are localised in the left well, the state $|L \uparrow, L \uparrow\rangle$ is not entangled, whereas, the state $|L \uparrow, L \downarrow\rangle$ is maximally entangled 
analogous to the Bell state for distinguishable qubits. In arriving at this result the one-particle basis used is finite-dimensional, because only the spin degrees of freedom of the qubits are considered.

In our model, the states $\left|\Phi_{0,0}\right\rangle$ and $\left|\Phi_{1,0}\right\rangle$ are analogous to the states $|L \uparrow, L \uparrow\rangle$ and $|L \uparrow, L \downarrow\rangle$. But there are crucial differences. The states in our model represent not two indistinguishable qubits, but two indistinguishable particles. This has important ramifications.

First, the entropy need not be bounded by unity. Second, it depends on the statistics parameter $\eta$. That is what is displayed in the above plots. From these one can read off the approximate values of the entropy obtained using numerical analysis for any given value of $\eta$.

It is interesting to note that in spite of these differences our results agree with [10] in the limiting cases of $\eta \rightarrow 0$ and $\eta \rightarrow \infty$, corresponding to bosons and fermions respectively. To understand this one has to look at the non-vanishing eigenvalues of the reduced density matrices. However, one has to remember that the two systems are really physically very different. A subtle point to note is that, as already pointed out, the states $\left|\Phi_{0,0}\right\rangle$ and $\left|\Phi_{1,0}\right\rangle$ are analogous to the states $|L \uparrow, L \uparrow\rangle$ and $|L \uparrow, L \downarrow\rangle$ respectively. To be more precise, as $\eta \rightarrow 0$, namely the bosonic limit, the state $\left|\Phi_{1,0}\right\rangle$ is entangled, so is the bosonic state $|L \uparrow, L \downarrow\rangle$. As $\eta \rightarrow \infty$, namely the fermionic limit, the state $\left|\Phi_{1,0}\right\rangle$ is entangled, so is the fermionic state $|L \uparrow, L \downarrow\rangle$. As $\eta \rightarrow 0$, the state $\left|\Phi_{0,0}\right\rangle$ is entangled, so is the bosonic state $|L \uparrow, L \uparrow\rangle$. Finally as $\eta \rightarrow \infty$, the state $\left|\Phi_{0,0}\right\rangle$ vanishes as already explained, and the fermionic state $|L \uparrow, L \uparrow\rangle$ is identically zero due to Pauli's exclusion principle. Hence qualitatively, the two systems appear to be identical in these limits if we formally identify the spin degrees of freedom of the qubit with the two levels labeling the $\Phi_{j, i}$.

The other results that Lo Franco and Compagno obtain regarding non-local entanglement use superpositions of states localised in the left and right wells, and are beyond the scope of the present work. 


\section{Conclusions}

The problem of studying the entanglement between indistinguishable particles in quantum mechanics is tricky. A naive usage of the usual measures like the Schmidt rank and the von Neumann entropy leads to wrong results.

A way to bypass these problems, restricted to bosons and fermions, was developed by Lo Franco and Compagno [10] by using ideas coming from information theory.

In this paper we use their results, in the second quantized formulation, to study the entanglement between two one-dimensional anyons. The generalised algebra of one-dimensional anyons obtained from a second quantization of the Leinaas-Myrheim model [18 plays a crucial role in our analysis.

We succeed in obtaining both qualitative and approximately quantitative results for the dependence of the von Neumann entropy on the statistics parameter.

The calculations presented in this paper are readily generalizable to studying entanglement between two clusters of anyons with an arbitrary number of particles. Other one-dimensional models admitting anyonic statistics like indistinguishable particles on a ring and the Calogero model are also worth investigating.

The most interesting problem will, of course, be to investigate the entanglement between anyons in two dimensions, both in the abelian and non-abelian cases, because of their direct relevance to topological quantum computation.

We hope to address these questions in our future work.

\section{Acknowledgments}

We thank Alok Laddha for discussions and the referees for many useful suggestions. This work is partially supported by a grant to CMI from the Infosys Foundation. 


\section{A The reduced density matrix in the second quantization formalism}

In the second quantization language the $N$-particle state $|\Phi\rangle$ is obtained by acting with a suitable combination of creation operators on the vacuum state. Let the set of states $\left\{\left|\psi_{k}\right\rangle \equiv a_{\psi_{k}}^{\dagger}|0\rangle\right\}$ form a basis for the single particle Hilbert space. In analogy with equation 3 , the state $\left|\phi_{k}\right\rangle$ is defined in the second quantization formalism as follows

$$
\left|\phi_{k}\right\rangle=\frac{a_{\psi_{k}}|\Phi\rangle}{\sqrt{\left\langle\Phi\left|a_{\psi_{k}}^{\dagger} a_{\psi_{k}}\right| \Phi\right\rangle}}
$$

The corresponding probabilities are

$$
p_{k}=\frac{\left\langle\Phi\left|a_{\psi_{k}}^{\dagger} a_{\psi_{k}}\right| \Phi\right\rangle}{\langle\Phi|\hat{\mathbf{n}}| \Phi\rangle}
$$

where $\hat{\mathbf{n}}=\sum_{k} a_{\psi_{k}}^{\dagger} a_{\psi_{k}}$ is the total number operator. Then, the one-particle reduced density matrix is

$$
\rho^{(1)}=\frac{\sum_{k} a_{\psi_{k}}|\Phi\rangle\langle\Phi| a_{\psi_{k}}^{\dagger}}{\langle\Phi|\hat{\mathbf{n}}| \Phi\rangle}
$$

\section{B Real space algebra}

\section{B.1 Derivation of the real space algebra}

The algebra of creation and annihilation operators of momentum states obtained in $[18]$ is

$$
\begin{aligned}
a_{p}^{\dagger} a_{q}^{\dagger} & =e^{i \phi_{\eta}(p-q)} a_{q}^{\dagger} a_{p}^{\dagger} \\
a_{p} a_{q}^{\dagger} & =e^{-i \phi_{\eta}(p-q)} a_{q}^{\dagger} a_{p}+\delta(p-q)
\end{aligned}
$$

where the phase $e^{i \phi_{\eta}(p-q)}=\frac{p-q+i \eta}{p-q-i \eta}$. The above relations may be rewritten in a slightly modified way as follows

$$
\begin{aligned}
a_{p}^{\dagger} a_{q}^{\dagger} & =\left(\frac{p-q+i \eta}{p-q-i \eta}\right) a_{q}^{\dagger} a_{p}^{\dagger} \\
a_{p} a_{q}^{\dagger} & =\left(\frac{p-q-i \eta}{p-q+i \eta}\right) a_{q}^{\dagger} a_{p}+\delta(p-q)
\end{aligned}
$$


Note that the creation and annihilation operators for the momentum states are related to the second quantized fields through the relations

$$
\Psi^{\dagger}(x)=\frac{1}{\sqrt{2 \pi}} \int_{-\infty}^{\infty} d k e^{i k x} a_{k}^{\dagger}
$$

To obtain the algebra of field operators we calculate the commutator between field operators

$$
\Psi(x) \Psi^{\dagger}(y)-\Psi^{\dagger}(y) \Psi(x)=\int_{-\infty}^{\infty} d p d q e^{-i p x+i q y}\left(a_{p} a_{q}^{\dagger}-a_{q}^{\dagger} a_{p}\right)
$$

Substituting for $a_{p} a_{q}^{\dagger}$ from the algebra of creation and annihilation operators for momentum states,

$$
\begin{aligned}
\Psi(x) \Psi^{\dagger}(y) & -\Psi^{\dagger}(y) \Psi(x) \\
& =\frac{1}{2 \pi} \int_{-\infty}^{\infty} d p d q e^{-i p x+i q y} a_{q}^{\dagger} a_{p}\left(\left(\frac{p-q-i \eta}{p-q+i \eta}\right)-1\right)+\delta(p-q) \\
& =\delta(x-y)-\frac{\eta}{\pi} \int_{-\infty}^{\infty} d p d q e^{-i p x+i q y} a_{q}^{\dagger} a_{p}\left(\frac{1}{-i p+i q+\eta}\right) \\
& =\delta(x-y)-\frac{\eta}{\pi} \int_{0}^{\infty} d z e^{-z \eta} \int_{-\infty}^{\infty} d p d q e^{-i p(x-z)+i q(y-z)} a_{q}^{\dagger} a_{p} \\
& =\delta(x-y)-2 \eta \int_{0}^{\infty} d z e^{-z \eta} \Psi^{\dagger}(y-z) \Psi(x-z)
\end{aligned}
$$

Similarly, if we look at the commutator $\left[\Psi^{\dagger}(x), \Psi^{\dagger}(y)\right]$, we obtain

$$
\Psi^{\dagger}(x) \Psi^{\dagger}(y)-\Psi^{\dagger}(y) \Psi^{\dagger}(x)=\int_{-\infty}^{\infty} d p d q e^{i p x+i q y}\left(a_{p}^{\dagger} a_{q}^{\dagger}-a_{q}^{\dagger} a_{p}^{\dagger}\right)
$$

Substituting for $a_{p}^{\dagger} a_{q}^{\dagger}$,

$$
\begin{aligned}
\Psi^{\dagger}(x) \Psi^{\dagger}(y) & -\Psi^{\dagger}(y) \Psi^{\dagger}(x) \\
& =\frac{1}{2 \pi} \int_{-\infty}^{\infty} d p d q e^{i p x+i q y} a_{q}^{\dagger} a_{p}^{\dagger}\left(\left(\frac{p-q+i \eta}{p-q-i \eta}\right)-1\right) \\
& =-\frac{\eta}{\pi} \int_{-\infty}^{\infty} d p d q e^{i p x+i q y} a_{q}^{\dagger} a_{p}^{\dagger} \int_{0}^{\infty} d z e^{-z(i p-i q+\eta)} \\
& =-\frac{\eta}{\pi} \int_{0}^{\infty} d z e^{-z \eta} \int_{-\infty}^{\infty} d p d q e^{i p(x-z)+i q(y+z)} a_{q}^{\dagger} a_{p}^{\dagger} \\
& =-2 \eta \int_{0}^{\infty} d z e^{-z \eta} \Psi^{\dagger}(y+z) \Psi^{\dagger}(x-z)
\end{aligned}
$$


Instead if we substitute for $a_{q}^{\dagger} a_{p}^{\dagger}$,

$$
\begin{aligned}
\Psi^{\dagger}(x) \Psi^{\dagger}(y)-\Psi^{\dagger}(y) \Psi^{\dagger}(x) & =\int_{-\infty}^{\infty} d p d q e^{i p x+i q y}\left(a_{p}^{\dagger} a_{q}^{\dagger}-a_{q}^{\dagger} a_{p}^{\dagger}\right) \\
& =\int_{-\infty}^{\infty} d p d q e^{i p x+i q y} a_{p}^{\dagger} a_{q}^{\dagger}\left(\frac{2 i \eta}{p-q+i \eta}\right) \\
& =2 \eta \int_{-\infty}^{\infty} d p d q e^{i p x+i q y} a_{p}^{\dagger} a_{q}^{\dagger} \int_{0}^{\infty} d z e^{-z(-i p+i q+\eta)} \\
& =2 \eta \int_{0}^{\infty} d z e^{-z \eta} \Psi^{\dagger}(x+z) \Psi^{\dagger}(y-z)
\end{aligned}
$$

\section{B.2 Commutation relations involving number operator}

The number operators is $\hat{N}=\int_{-\infty}^{\infty} d x \Psi^{\dagger}(x) \Psi(x)$. We calculate the commutator between the number operator and the field theoretic anyon creation operator by substituting in terms of momentum space operators as follows

$$
\begin{aligned}
{\left[\hat{N}, \Psi^{\dagger}(y)\right] } & =\int_{-\infty}^{\infty} d x\left[\Psi^{\dagger}(x) \Psi(x), \Psi^{\dagger}(y)\right] \\
& =\frac{1}{(2 \pi)^{\frac{3}{2}}} \int_{-\infty}^{\infty} d x \int_{-\infty}^{\infty} d p d q d r e^{i p x-i q x+i r y}\left(a_{p}^{\dagger} a_{q} a_{r}^{\dagger}-a_{r}^{\dagger} a_{p}^{\dagger} a_{q}\right) \\
& =\frac{1}{(2 \pi)^{\frac{3}{2}}} \int_{-\infty}^{\infty} d p d r e^{i r y}\left(a_{p}^{\dagger} a_{p} a_{r}^{\dagger}-a_{r}^{\dagger} a_{p}^{\dagger} a_{p}\right) \\
& =\frac{1}{(2 \pi)^{\frac{3}{2}}} \int_{-\infty}^{\infty} d p d r e^{i r y}\left(a_{p}^{\dagger}\left(e^{-i \phi_{\eta}(p-r)} a_{r}^{\dagger} a_{p}+\delta(p-r)\right)-a_{r}^{\dagger} a_{p}^{\dagger} a_{p}\right) \\
& =\frac{1}{(2 \pi)^{\frac{3}{2}}} \int_{-\infty}^{\infty} d p d r e^{i r y}\left(\delta(p-r)+e^{-i \phi_{\eta}(p-r)} e^{i \phi_{\eta}(p-r)} a_{r}^{\dagger} a_{p}^{\dagger} a_{p}-a_{r}^{\dagger} a_{p}^{\dagger} a_{p}\right) \\
& =\Psi^{\dagger}(y)
\end{aligned}
$$

The corresponding result for the annihilation operator is

$$
[\hat{N}, \Psi(y)]=-\Psi(y)
$$

The same results can be obtained using the real space operator algebra as shown below

$$
\begin{aligned}
{\left[\hat{N}, \Psi^{\dagger}(y)\right] } & =\int_{-\infty}^{\infty} d x\left(\Psi^{\dagger}(x) \Psi(x) \Psi^{\dagger}(y)-\Psi^{\dagger}(y) \Psi^{\dagger}(x) \Psi(x)\right) \\
& =\int_{-\infty}^{\infty} d x\left(\Psi ^ { \dagger } ( x ) \left(\Psi^{\dagger}(y) \Psi(x)+\delta(x-y)\right.\right.
\end{aligned}
$$




$$
\begin{aligned}
- & \left.\left.2 \eta \int_{0}^{\infty} d z e^{-z \eta} \Psi^{\dagger}(y-z) \Psi(x-z)\right)-\Psi^{\dagger}(y) \Psi^{\dagger}(x) \Psi(x)\right) \\
= & \Psi^{\dagger}(y) \\
+ & \int_{-\infty}^{\infty} d x\left(\left(\left(\Psi^{\dagger}(y) \Psi^{\dagger}(x)+2 \eta \int_{0}^{\infty} d z e^{-z \eta} \Psi^{\dagger}(x+z) \Psi^{\dagger}(y-z)\right) \Psi(x)\right.\right. \\
& \left.\left.-2 \eta \int_{0}^{\infty} d z e^{-z \eta} \Psi^{\dagger}(x) \Psi^{\dagger}(y-z) \Psi(x-z)\right)-\Psi^{\dagger}(y) \Psi^{\dagger}(x) \Psi(x)\right) \\
= & \Psi^{\dagger}(y)
\end{aligned}
$$

With a similar calculation, we can obtain the commutator of the number operator with field theoretic anyon annihilation operator.

\section{B.3 Check on the algebra}

The symmetrization postulate for multiparticle wave functions in the first quantized formalism has an intimate connection with the algebra of creation and annihilation operators in the second quantized formalism. This was clearly explained in very general terms by Fock for the case of bosons and fermions in 22. In this appendix we verify the consistency of the anyonic algebra we use along similar lines.

The field operator $\Psi(x)$ acts on the sequence of functions

$$
\left(\begin{array}{c}
\text { const. } \\
\psi\left(x_{1}\right) \\
\psi\left(x_{1}, x_{2}\right) \\
\psi\left(x_{1}, x_{2}, x_{3}\right) \\
\cdots \cdots
\end{array}\right)
$$

as follows

$$
\Psi(x)\left(\begin{array}{c}
\text { const. } \\
\psi\left(x_{1}\right) \\
\psi\left(x_{1}, x_{2}\right) \\
\psi\left(x_{1}, x_{2}, x_{3}\right) \\
\ldots \ldots
\end{array}\right)=\left(\begin{array}{c}
\psi(x) \\
\sqrt{2} \psi\left(x, x_{1}\right) \\
\sqrt{3} \psi\left(x, x_{1}, x_{2}\right) \\
\sqrt{4} \psi\left(x, x_{1}, x_{2}, x_{3}\right) \\
\ldots \ldots \ldots
\end{array}\right)
$$

where the functions $\psi\left(x_{1}\right), \psi\left(x_{1}, x_{2}\right), \psi\left(x_{1}, x_{2}, x_{3}\right) \ldots$ are interpreted as Schrodinger wave functions 22 . 
Applying the operator $\Psi\left(x^{\prime}\right) \Psi(x)$ on the sequence of functions, we obtain

$$
\Psi\left(x^{\prime}\right) \Psi(x)\left(\begin{array}{c}
\text { const. } \\
\psi\left(x_{1}\right) \\
\psi\left(x_{1}, x_{2}\right) \\
\psi\left(x_{1}, x_{2}, x_{3}\right) \\
\cdots \ldots
\end{array}\right)=\left(\begin{array}{c}
\sqrt{2.1} \psi\left(x, x^{\prime}\right) \\
\sqrt{3.2} \psi\left(x, x^{\prime}, x_{1}\right) \\
\sqrt{4.3} \psi\left(x, x^{\prime}, x_{1}, x_{2}\right) \\
\sqrt{5.4} \psi\left(x, x^{\prime}, x_{1}, x_{2}, x_{3}\right) \\
\ldots \ldots
\end{array}\right)
$$

Similarly, applying the operator $\Psi(x) \Psi\left(x^{\prime}\right)$ on the sequence of functions, we get

$$
\Psi(x) \Psi\left(x^{\prime}\right)\left(\begin{array}{c}
\text { const. } \\
\psi\left(x_{1}\right) \\
\psi\left(x_{1}, x_{2}\right) \\
\psi\left(x_{1}, x_{2}, x_{3}\right) \\
\cdots \cdots
\end{array}\right)=\left(\begin{array}{c}
\sqrt{2.1} \psi\left(x^{\prime}, x\right) \\
\sqrt{3.2} \psi\left(x^{\prime}, x, x_{1}\right) \\
\sqrt{4.3} \psi\left(x^{\prime}, x, x_{1}, x_{2}\right) \\
\sqrt{5.4} \psi\left(x^{\prime}, x, x_{1}, x_{2}, x_{3}\right) \\
\cdots \cdots
\end{array}\right)
$$

In the case of bosons, the right hand side of Eq24 and Eq25 are the same because the bosonic wave function is symmetric under the exchange of any pair of coordinates. This implies that the field operators $\Psi(x)$ and $\Psi\left(x^{\prime}\right)$ commute with each other. In the case of fermions, using the same argument and by noting that the fermionic wave functions are anti-symmetric, one can obtain the usual anti-commutation relation between $\Psi(x)$ and $\Psi\left(x^{\prime}\right)$.

In our case the field operators satisfy the following algebra

$$
\begin{aligned}
{\left[\Psi(x), \Psi^{\dagger}(y)\right] } & =\delta(x-y)-2 \eta \int_{0}^{\infty} d z e^{-z \eta} \Psi^{\dagger}(y-z) \Psi(x-z) \\
{\left[\Psi^{\dagger}(x), \Psi^{\dagger}(y)\right] } & =-2 \eta \int_{0}^{\infty} d z e^{-z \eta} \Psi^{\dagger}(y+z) \Psi^{\dagger}(x-z)
\end{aligned}
$$

The consistency of the algebra requires that the following equation holds

$$
\left.\left(\Psi(x) \Psi(y)-\Psi(y) \Psi(x)-2 \eta \int_{0}^{\infty} d z e^{-z \eta} \Psi(x-z) \Psi(y+z)\right)\right)\left(\begin{array}{c}
\text { const. } \\
\psi\left(x_{1}\right) \\
\psi\left(x_{1}, x_{2}\right) \\
\psi\left(x_{1}, x_{2}, x_{3}\right) \\
\ldots \ldots . . .
\end{array}\right)=0
$$

ie,

$$
\left(\begin{array}{c}
\sqrt{2.1}\left(\psi(y, x)-\psi(x, y)-2 \eta \int_{0}^{\infty} d z e^{-z \eta} \psi(y+z, x-z)\right) \\
\sqrt{3.2}\left(\psi\left(y, x, x_{1}\right)-\psi\left(x, y, x_{1}\right)-2 \eta \int_{0}^{\infty} d z e^{-z \eta} \psi\left(y+z, x-z, x_{1}\right)\right) \\
\sqrt{4.3}\left(\psi\left(y, x, x_{1}, x_{2}\right)-\psi\left(x, y, x_{1}, x_{2}\right)-2 \eta \int_{0}^{\infty} d z e^{-z \eta} \psi\left(y+z, x-z, x_{1}, x_{2}\right)\right) \\
\cdots \ldots . .
\end{array}\right)=0
$$


where $\psi\left(x_{1}, x_{2}, . ., x_{N}\right)$ is the $N$-anyon wave function. Let the wave function be

$$
\psi\left(x_{1}, . ., x_{N}\right)=\sum_{P \in S_{N}} \alpha\left(k_{P(1)}, . ., k_{P(N)}\right) e^{i\left(k_{P(1)} x_{1}+\ldots+k_{P(N)} x_{N}\right)}
$$

where the coefficients satisfy

$$
\alpha\left(\ldots k_{j}, . . k_{l}, . .\right)=\left(\frac{k_{j}-k_{l}-i \eta}{k_{j}-k_{l}+i \eta}\right) \alpha\left(\ldots k_{l}, . . k_{i}, . .\right)
$$

We have to calculate

$$
\left(\psi\left(y, x, x_{3}, . ., x_{N}\right)-\psi\left(x, y, x_{3}, . ., x_{N}\right)-2 \eta \int_{0}^{\infty} d z e^{-z \eta} \psi\left(y+z, x-z, x_{3}, . ., x_{N}\right)\right)
$$

Substituting the expression for the wave function

$$
\begin{aligned}
\sum_{P \in S_{N}} & \left(\alpha\left(k_{P(1)}, . ., k_{P(N)}\right) e^{i\left(k_{P(1)} y+k_{P(2)} x+k_{P(3)} x_{3}+\ldots+k_{P(N)} x_{N}\right)}\right. \\
& -\alpha\left(k_{P(1)}, . ., k_{P(N)}\right) e^{i\left(k_{P(1)} x+k_{P(2)} y+k_{P(3)} x_{3}+\ldots+k_{P(N)} x_{N}\right)} \\
& \left.-2 \eta \int_{0}^{\infty} d z e^{-z \eta} \alpha\left(k_{P(1)}, . ., k_{P(N)}\right) e^{i\left(k_{P(1)}(y+z)+k_{P(2)}(x-z)+k_{P(3)} x_{3}+\ldots+k_{P(N)} x_{N}\right)}\right) \\
& =\sum_{P \in S_{N}}\left(\alpha\left(k_{P(1)}, . ., k_{P(N)}\right) e^{i\left(k_{P(1)} y+k_{P(2)} x+k_{P(3)} x_{3}+\ldots+k_{P(N)} x_{N}\right)}\right. \\
& -\alpha\left(k_{P(1)}, . ., k_{P(N)}\right) e^{i\left(k_{P(1)} x+k_{P(2)} y+k_{P(3)} x_{3}+\ldots+k_{P(N)} x_{N}\right)} \\
& \left.-\frac{2 i \eta}{k_{P(1)}-k_{P(2)}+i \eta} \alpha\left(k_{P(1)}, . ., k_{P(N)}\right) e^{i\left(k_{P(1)} y+k_{P(2)} x+k_{P(3)} x_{3}+\ldots+k_{P(N)} x_{N}\right)}\right)
\end{aligned}
$$

we find that the coefficient of the term $e^{i\left(k_{P(1)} x+k_{P(2)} y+k_{P(3)} x_{3}+\ldots+k_{P(N)} x_{N}\right)}$ is

$$
\alpha\left(k_{P(2)}, k_{P(1)}, . ., k_{P(N)}\right)-\left(\frac{k_{P(1)}-k_{P(2)}-i \eta}{k_{P(1)}-k_{P(2)}+i \eta}\right) \alpha\left(k_{P(1)}, k_{P(2)}, . ., k_{P(N)}\right)
$$

Using the relation among coefficients, it is easy to see that above term is zero, as expected.

\section{Calculation of the one-particle reduced den- sity matrix}

The matrix elements of the one-particle reduced density matrix are

$$
\rho_{m, n}^{(1)}=\frac{\sum_{k=0}^{\infty}\left\langle 0\left|\Psi_{h_{m}} \Psi_{h_{k}}\right| \Phi_{j, i}\right\rangle\left\langle\Phi_{j, i}\left|\Psi_{h_{k}}^{\dagger} \Psi_{h_{n}}^{\dagger}\right| 0\right\rangle}{\left\langle\Phi_{j, i}|\hat{\mathbf{n}}| \Phi_{j, i}\right\rangle}
$$


Using the definition of the state $\left|\Phi_{j, i}\right\rangle$, it is rewritten as,

$$
\rho_{m n}^{(1)}=\frac{\sum_{k}\left\langle 0\left|\Psi_{h_{m}} \Psi_{h_{k}} \Psi_{h_{j}}^{\dagger} \Psi_{h_{i}}^{\dagger}\right| 0\right\rangle\left\langle 0\left|\Psi_{h_{i}} \Psi_{h_{j}} \Psi_{h_{k}}^{\dagger} \Psi_{h_{n}}^{\dagger}\right| 0\right\rangle}{2\left\langle 0\left|\Psi_{h_{i}} \Psi_{h_{j}} \Psi_{h_{j}}^{\dagger} \Psi_{h_{i}}^{\dagger}\right| 0\right\rangle}
$$

To obtain the expression for the one-particle reduced density matrix a generic term of the following form is calculated

$$
\begin{aligned}
\left\langle 0\left|\Psi_{h_{m}} \Psi_{h_{k}} \Psi_{h_{j}}^{\dagger} \Psi_{h_{i}}^{\dagger}\right| 0\right\rangle & =\left\langle h_{k} \mid h_{j}\right\rangle\left\langle h_{m} \mid h_{i}\right\rangle+\left\langle h_{k} \mid h_{i}\right\rangle\left\langle h_{m} \mid h_{j}\right\rangle \\
& -\int_{0}^{\infty} d z 2 \eta e^{-z \eta} \int_{-\infty}^{\infty} d x d y h_{m}^{*}(y-z) h_{k}^{*}(x) h_{j}(y) h_{i}(x-z)
\end{aligned}
$$

Using the above formula, the denominator of the one-particle reduced density matrix can be obtained by setting $m=i$ and $k=j$. The numerator is calculated below.

$$
\begin{aligned}
\sum_{k}\left\langle 0\left|\Psi_{h_{m}} \Psi_{h_{k}} \Psi_{h_{j}}^{\dagger} \Psi_{h_{i}}^{\dagger}\right| 0\right\rangle\left\langle 0\left|\Psi_{h_{i}} \Psi_{h_{j}} \Psi_{h_{k}}^{\dagger} \Psi_{h_{n}}^{\dagger}\right| 0\right\rangle \\
=\left\langle h_{m} \mid h_{i}\right\rangle\left\langle h_{n} \mid h_{i}\right\rangle+\left\langle h_{i} \mid h_{j}\right\rangle\left\langle h_{m} \mid h_{i}\right\rangle\left\langle h_{n} \mid h_{j}\right\rangle \\
+\left\langle h_{j} \mid h_{i}\right\rangle\left\langle h_{m} \mid h_{j}\right\rangle\left\langle h_{i} \mid h_{n}\right\rangle+\left\langle h_{m} \mid h_{j}\right\rangle\left\langle h_{j} \mid h_{n}\right\rangle \\
-2 \eta\left\langle h_{m} \mid h_{i}\right\rangle \int_{0}^{\infty} d z \int_{-\infty}^{\infty} d x d y e^{-z \eta} h_{n}(y-z) h_{j}(x) h_{j}^{*}(y) h_{i}^{*}(x-z) \\
-2 \eta\left\langle h_{m} \mid h_{j}\right\rangle \int_{0}^{\infty} d z \int_{-\infty}^{\infty} d x d y e^{-z \eta} h_{n}(y-z) h_{i}(x) h_{j}^{*}(y) h_{i}^{*}(x-z) \\
-2 \eta\left\langle h_{i} \mid h_{n}\right\rangle \int_{0}^{\infty} d z \int_{-\infty}^{\infty} d x d y e^{-z \eta} h_{m}^{*}(y-z) h_{j}^{*}(x) h_{j}(y) h_{i}(x-z) \\
-2 \eta\left\langle h_{j} \mid h_{n}\right\rangle \int_{0}^{\infty} d z \int_{-\infty}^{\infty} d x d y e^{-z \eta} h_{m}^{*}(y-z) h_{i}^{*}(x) h_{j}(y) h_{i}(x-z) \\
+4 \eta^{2} \int_{0}^{\infty} d z d z^{\prime} \int_{-\infty}^{\infty} d x d y d y^{\prime}\left(e^{-\left(z+z^{\prime}\right) \eta} h_{m}^{*}(y-z) h_{j}(y) h_{i}(x-z)\right. \\
\left.\times h_{n}\left(y^{\prime}-z^{\prime}\right) h_{j}^{*}\left(y^{\prime}\right) h_{i}^{*}\left(x-z^{\prime}\right)\right)
\end{aligned}
$$

To calculate the one-particle reduced density matrix, we use the following integrals [24].

$$
\begin{aligned}
& \int_{-\infty}^{\infty} d z e^{-\frac{z^{2}}{2}-\frac{1}{2}(z-\zeta)^{2}} H_{n}(z) H_{p}(z-\zeta) \\
& \quad=\frac{1}{\Gamma(n+1)} \sqrt{\pi} e^{-\frac{\zeta^{2}}{4}} \sqrt{2^{n} n !} \sqrt{2^{p} p !}(-\zeta)^{p-n}
\end{aligned}
$$




$$
\begin{gathered}
\times \sqrt{2^{n-p} \Gamma(n+1) \Gamma(p+1)}{ }_{1} \tilde{F}_{1}\left(-n ;-n+p+1 ; \frac{\zeta^{2}}{2}\right) \quad, n, p \in \mathbb{N} \\
\int_{0}^{\infty} x^{\nu-1} e^{-\beta x^{2}-\gamma x}=(2 \beta)^{-\frac{\nu}{2}} \Gamma(\nu) e^{\frac{\gamma^{2}}{8 \beta}} D_{-\nu}\left(\frac{\gamma}{\sqrt{2 \beta}}\right), \nu>-1
\end{gathered}
$$

Here ${ }_{1} \tilde{F}_{1}(a ; b ; z)$ denotes the regularized confluent hypergeometric function and $D_{-\nu}(z)$ denotes the parabolic cylinder function. The matrix elements of the oneparticle reduced density matrix obtained from the initial state $\left|\Phi_{0,0}\right\rangle$ are given below.

$$
\begin{aligned}
\left(\rho_{0,0}^{(1)}\right)_{m, n}= & \frac{1}{d_{1}}\left(4 \delta_{m 0} \delta_{n 0}-4 \eta \delta_{m 0}(-1)^{n} \frac{1}{\sqrt{2^{n} n !}} \mathfrak{D}(n+1, \eta)\right. \\
& -4 \eta \delta_{n 0}(-1)^{m} \frac{1}{\sqrt{2^{m} m !}} \mathfrak{D}(m+1, \eta) \\
& \left.+(-1)^{m+n} \frac{4 \eta^{2}}{\sqrt{2^{m+n} m ! n !}} \sum_{l=0}^{\infty}\left(\frac{1}{2^{l} l !} \mathfrak{D}(m+l+1, \eta) \mathfrak{D}(n+l+1, \eta)\right)\right)
\end{aligned}
$$

where $\mathfrak{D}(\nu, x)=\Gamma(\nu) e^{\frac{\eta^{2}}{4}} D_{-\nu}(x)$ and

$$
d_{1}=4(1-\eta \mathfrak{D}(-1, \eta))
$$

The matrix elements of the one-particle reduced density matrix obtained from the initial state $\left|\Phi_{1,0}\right\rangle$ are given below

$$
\begin{aligned}
\left(\rho_{1,0}^{(1)}\right)_{m, n}= & \frac{1}{d_{2}}\left(\delta_{m 1} \delta_{n 1}+\delta_{m 0} \delta_{n 0}-\delta_{m 1} \frac{(-1)^{n+1} \sqrt{2} \eta}{\sqrt{2^{n} n !}} \mathfrak{D}(n+2, \eta)\right. \\
& -\delta_{m 0} \frac{\eta(-1)^{n}}{\sqrt{2^{n} n !}}(2 \mathfrak{D}(n+1, \eta)-\mathfrak{D}(n+3, \eta)) \\
& -\delta_{n 1} \frac{(-1)^{m+1} \sqrt{2} \eta}{\sqrt{2^{m} m !}} \mathfrak{D}(m+2, \eta) \\
& -\delta_{n 0} \frac{\eta(-1)^{m}}{\sqrt{2^{m} m !}}(2 \mathfrak{D}(m+1, \eta)-\mathfrak{D}(m+3, \eta)) \\
& +\frac{4 \eta^{2}(-1)^{m+n}}{2^{m+n} m ! n !} \sum_{l=0}^{\infty} \frac{1}{2^{l} l !}(2 \mathfrak{D}(n+l+1, \eta) \mathfrak{D}(m+l+1, \eta) \\
& -\mathfrak{D}(n+l+1, \eta) \mathfrak{D}(m+l+3, \eta)+2 \mathfrak{D}(n+l+2, \eta) \mathfrak{D}(m+l+2 \eta) \\
& -\mathfrak{D}(n+l+3, \eta) \mathfrak{D}(m+l+1, \eta)))
\end{aligned}
$$

where

$$
d_{2}=2\left(1+\frac{\eta}{2} \mathfrak{D}(3, \eta)\right)
$$




\section{References}

[1] Frank Wilczek. Fractional statistics and anyon superconductivity, volume 5. World scientific, 1990.

[2] Avinash Khare. Fractional statistics and quantum theory. World Scientific, 2005.

[3] Alexei Kitaev. Anyons in an exactly solved model and beyond. Annals of Physics, 321(1):2-111, 2006.

[4] John Preskill. Battling decoherence: The fault-tolerant quantum computer. Physics Today, 52(6):24-30, Jun 1999.

[5] Sankar Das Sarma, Michael Freedman, and Chetan Nayak. Topological quantum computation. Physics Today, 59(7):32-38, 2006.

[6] AP Balachandran, TR Govindarajan, Amilcar R de Queiroz, and AF Reyes-Lega. Algebraic approach to entanglement and entropy. Physical Review A, 88(2):022301, 2013.

[7] GianCarlo Ghirardi and Luca Marinatto. Identical particles and entanglement. Optics and Spectroscopy, 99(3):386-390, 2005.

[8] John Schliemann, J Ignacio Cirac, Marek Kuś, Maciej Lewenstein, and Daniel Loss. Quantum correlations in two-fermion systems. Physical Review A, 64(2):022303, 2001.

[9] R Paškauskas and L You. Quantum correlations in two-boson wave functions. Physical Review A, 64(4):042310, 2001.

[10] Rosario Lo Franco and Giuseppe Compagno. Quantum entanglement of identical particles by standard information-theoretic notions. Scientific reports, 6:20603, 2016. 
[11] Howard Mark Wiseman and John A Vaccaro. Entanglement of indistinguishable particles shared between two parties. Physical review letters, 91(9):097902, 2003.

[12] N Killoran, M Cramer, and Martin B Plenio. Extracting entanglement from identical particles. Physical review letters, 112(15):150501, 2014.

[13] Rosario Lo Franco and Giuseppe Compagno. Indistinguishability of elementary systems as a resource for quantum information processing. Physical review letters, 120(24):240403, 2018.

[14] Fabio Benatti, Roberto Floreanini, and Kelvin Titimbo. Entanglement of identical particles. Open Systems $\&$ Information Dynamics, 21(01n02):1440003, 2014.

[15] Giuseppe Compagno, Alessia Castellini, and Rosario Lo Franco. Dealing with indistinguishable particles and their entanglement. Philosophical Transactions of the Royal Society A: Mathematical, Physical and Engineering Sciences, 376(2123):20170317, 2018.

[16] Antônio C Lourenço, Tiago Debarba, and Eduardo I Duzzioni. Entanglement of indistinguishable particles: A comparative study. Physical Review A, 99(1):012341, 2019.

[17] Jon M Leinaas and Jan Myrheim. On the theory of identical particles. Il Nuovo Cimento B (1971-1996), 37(1):1-23, 1977.

[18] Thore Posske, Björn Trauzettel, and Michael Thorwart. Second quantization of leinaas-myrheim anyons in one dimension and their relation to the lieb-liniger model. Physical Review B, 96(19):195422, 2017.

[19] Albert Messiah. Quantum Mechanics Volume II. North Holland Publishing Company, 1967.

[20] Wolfgang Pauli. The connection between spin and statistics. Physical Review, 58(8):716, 1940. 
[21] Thore Posske, Björn Trauzettel, and Michael Thorwart. Second quantization of leinaas-myrheim anyons in one dimension and their relation to the lieb-liniger model. Physical Review B, 96(19):195422, 2017.

[22] Ludwig D Faddeev, LA Khalfin, and IV Komarov. VA Fock-selected works: Quantum mechanics and quantum field theory. CRC Press, 2004.

[23] Michael Reed. Methods of modern mathematical physics: Functional analysis. Elsevier, 2012.

[24] Izrail Solomonovich Gradshteyn and Iosif Moiseevich Ryzhik. Table of integrals, series, and products. Academic press, 2014. 